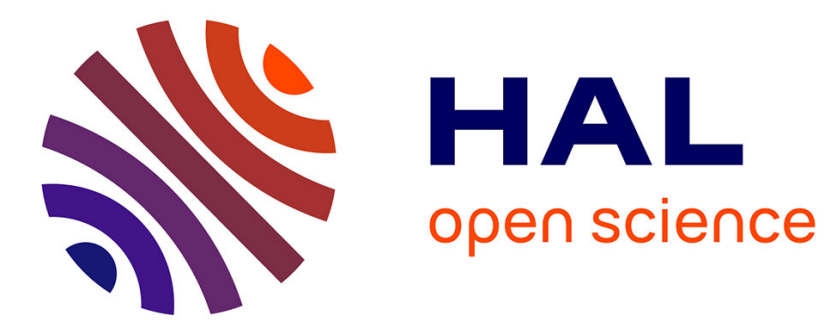

\title{
Des prénoms invisibles
}

Baptiste Coulmont

\section{To cite this version:}

Baptiste Coulmont. Des prénoms invisibles. La lettre de l'enfance et de l'adolescence, 2012, 87, pp.23-28. 10.3917/lett.087.0023 . halshs-01412153

\section{HAL Id: halshs-01412153 \\ https://shs.hal.science/halshs-01412153}

Submitted on 8 Dec 2016

HAL is a multi-disciplinary open access archive for the deposit and dissemination of scientific research documents, whether they are published or not. The documents may come from teaching and research institutions in France or abroad, or from public or private research centers.
L'archive ouverte pluridisciplinaire HAL, est destinée au dépôt et à la diffusion de documents scientifiques de niveau recherche, publiés ou non, émanant des établissements d'enseignement et de recherche français ou étrangers, des laboratoires publics ou privés. 


\title{
Des prénoms invisibles
}

\author{
Baptiste Coulmont
}

Références : Coulmont (Baptiste), «Des prénoms invisibles », La lettre de l'enfance et de l'adolescence, 201 2, n87, p.23-28 [DOI : 10.39I 7/lett.087.0023]

Le prénom porte publiquement une partie de l'identité de la personne : je m'appelle Baptiste. Mais vous ne saurez rien de mon second prénom : la plupart du temps, il est invisible. De même, si Baptiste était mon prénom d'usage, celui de mon état civil serait passé sous silence (mais c'est le prénom d'usage qui, inversement, serait invisible au regard de l'État). Deux « types» de prénoms qui ne se voient pas, donc, mais qui ne sont pas sans importance.

Les sociologues connaissent bien les premiers prénoms, qui sont souvent utilisés pour étudier la structure de la mode [Besnard, I979; Lieberson, 2000 ; Coulmont, $20 \mathrm{I}$ I]; beaucoup moins les seconds prénoms ou les prénoms d'usage absents de l'état civil. Explorons-les ici en quelques pages.

\section{Les seconds prénoms}

Pendant plusieurs siècles, après la fixation du binôme « nom de personne » « patronyme» au cours du Moyen Âge, les Français et Françaises nont porté qu'un seul prénom.

L'innovation est sans doute venue des nobles, à partir du XVIIe siècle, et s'est diffusée, lentement, au reste de la population [Perouas et al., I984, p. I 20 et suivantes]. Multiplier les prénoms avait sans doute plusieurs buts : placer l'enfant sous une multiplicité de saints-patrons, manifester la pluralité des lignées, augmenter les signes d'alliance en utilisant des prénoms de personnages prestigieux... mais aussi, et peut-être surtout, se différencier ainsi de ceux qui ne portaient qu'un seul prénom. Aujourd'hui encore certains prétendants au trône, en France, se distinguent par l'immensité de leur patrimoine prénominal. L'un des petits-fils du « comte de Paris » se nomme ainsi Aimone Umberto Emanuele Filiberto Luigi Amedeo Elena Maria Fiorenzo di Savoia-Aosta, «duc des Pouilles».

Donner plusieurs prénoms à ses enfants est une pratique qui se développe au cours du XIXe siècle : environ $45 \%$ des garçons et des filles naissent avec plus 
d'un prénom en I800, c'est le cas de 70\% des naissances vers I 900 [Dupâquier et al., I 987, p.28 et suivantes]. En Île de France, dès la fin du XVIIIe siècle déjà, $90 \%$ des enfants recevaient plus d'un prénom [Daumas, 2003, p.I 80 et suivantes]. La diffusion de cette pratique, en région, se fait parfois par l'intermédiaire des migrants, qui quittent leur village pour séjourner à Paris puis s'en retournent, avec d'autres manières de faire, là où ils sont nés : c'est du moins le cas en Limousin [Perouas et al., I984].

Les prénoms multiples ont contribué à l'élargissement du stock de prénoms : si le premier prénom était un prénom hérité (transmis par le parrain ou la marraine), les autres pouvaient être plus neufs, car abandonnés à l'imagination de celui ou celle qui était chargé de prénommer. Multiplier les prénoms permettait de garder la tradition (hériter d'un prénom) tout en faisant entrer l'innovation. Du point de vue de l'État, au contraire, les prénoms multiples permettaient d'identifier plus aisément des individus : si, dans un monde hypothétique, $20 \%$ des garçons s'appellent « Pierre», une proportion beaucoup plus petite s'appellera « Pierre, Simon».

Il est donc possible de comprendre les usages que les Français du XIX ${ }^{\mathrm{e}}$ siècle pouvaient faire des prénoms multiples. Qu'en est-il aujourd'hui ?

Il est difficile d'avoir accès à un échantillon représentatif de personnes vivant en France pour lequel tous les prénoms sont disponibles. Je vais donc ici m’appuyer, de manière exploratoire, sur les résultats au baccalauréat de 2008 et $20 \mathrm{I}$ I, des listes nominatives étant disponibles sur internet et comportant plusieurs centaines de milliers d'individus.

Les données ne permettent pas de savoir avec certitude quelle proportion des bacheliers de 20I I (nés autour de 1993) portent plus d'un prénom. Mais la pratique semble être en diminution depuis le XIXe siècle : il est vrai qu'aujourd'hui, le premier prénom, qui nest plus hérité ou transmis, individualise déjà fortement son porteur. Le prénom le plus donné, pour une année précise, rassemble rarement plus de $2 \%$ des naissances. Le temps est révolu où une fille sur cinq était baptisée «Marie».

A quoi donc peut bien servir une multiplicité de prénoms quand le premier prénom individualise déjà?

Un début de réponse est donné par les prénoms eux-mêmes : les « deuxièmes prénoms» les plus répandus sont, en 20 I I, Marie, Pierre, Jean, Michel, André, François, Claude, Louis, Paul, Anne, Jeanne, Philippe, Isabelle, Jacques, René, Françoise, Bernard, Dominique; c'est à dire des prénoms que l'on n'associe pas immédiatement à la génération de I 993. Les " premiers prénoms » les plus répandus chez les bacheliers de 20 I I sont Camille, Thomas, Marie, Marine, Maxime, Pauline, Marion, Laura, Alexandre, Manon, Nicolas, Kevin...

Il semble, à l'oreille, que les seconds prénoms soient des prénoms démodés. Et, comme je le précise ci-dessous, on en a confirmation statistiquement.

Quelle est lévolution de la popularité des cinquantes « deuxièmes prénoms» 
les plus répandus parmi les bacheliers de 20 i I ? Nous pouvons constater sur le graphique I - la courbe pointillée - que ces prénoms connaissent leur apogée (en tant que premiers prénoms) autour de I940, cinquante ans avant la naissance de ceux qui les portent, aujourd'hui, comme deuxièmes prénoms. " Manon, Françoise » a de grande chances d'être née au début des années I990; "Françoise », de son côté, est plutôt née à la fin des années quarante.

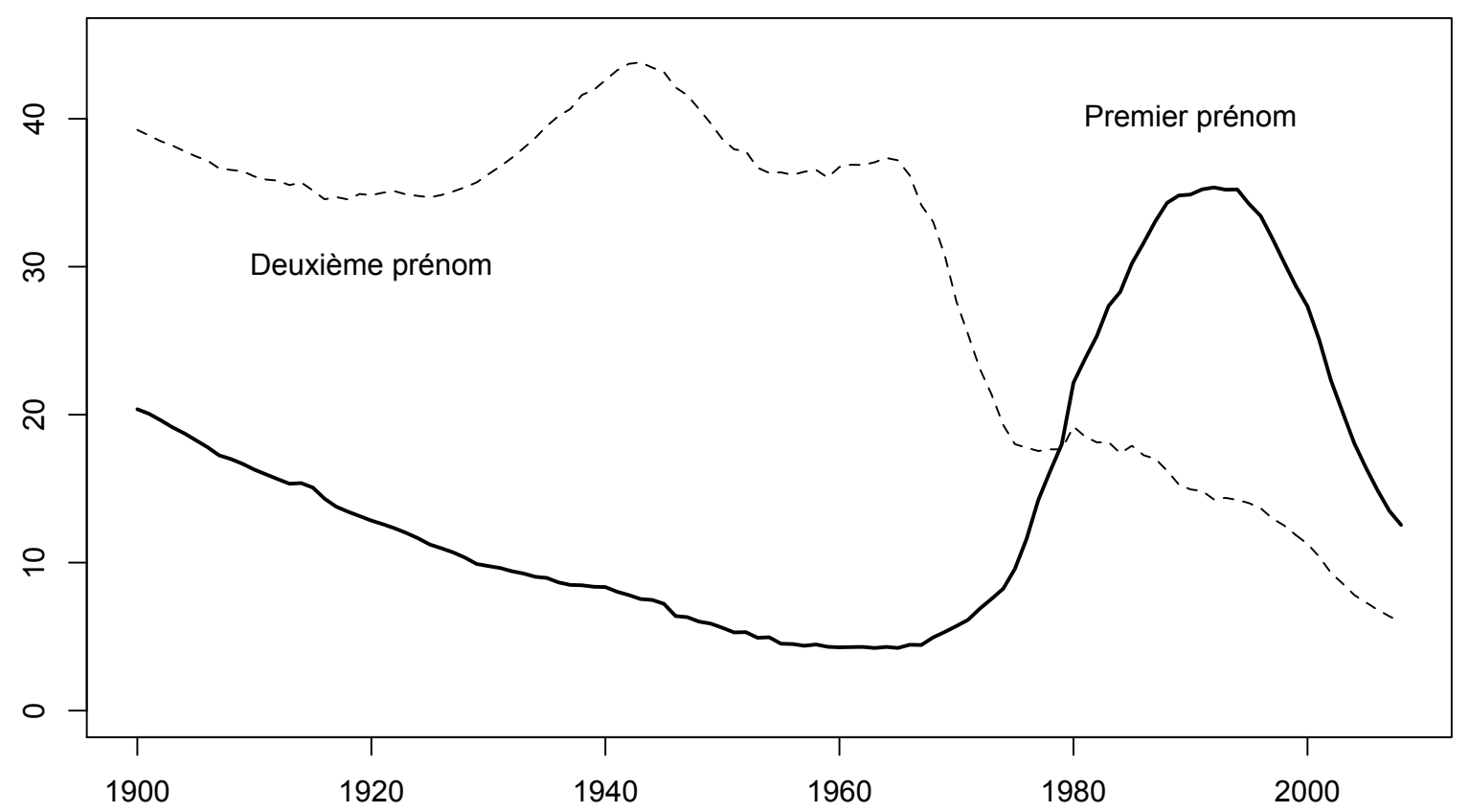

Fig. I : Évolution, au cours du $\mathrm{xx}^{\mathrm{e}}$ siècle, de la popularité des prénoms les plus fréquents chez les bacheliers de 20 I

(Lecture du graphique : En I 960, les 50 " premiers prénoms» les plus fréquents chez les bacheliers de 20 I I ont été donnés à $5 \%$ des naissances. La même année, les 50 " deuxièmes prénoms" les plus fréquents chez les mêmes bacheliers ont été donnés, en premier prénom, à près de $40 \%$ des naissances.)

On peut expliciter les choses d'une autre manière : parmi ceux des bacheliers de 20 I I qui portent un deuxième prénom, $56 \%$ portent un deuxième prénom dont le « pic de fréquence» est antérieur à 1970.

Alors que le premier prénom nous rattache directement au moment de notre naissance, les prénoms suivants ont tendance à nous attacher à des périodes antérieures : ils échappent à la logique de la mode (ou reprennent les modes passées). La multiplicité des prénoms, qui avait pu servir à introduire de l'innovation, avant la Révolution française, constitue aujourd'hui un conservatoire de la désuétude. En 
quelques siècles, un renversement a eu lieu : le premier prénom était hérité, il est désormais choisi par les parents et différent de leur prénom; le deuxième prénom, lui, semble désormais hérité. Mais est-ce le cas?

À ma connaissance, seul l'anthropologue Bernard Vernier [Vernier, I998; Vernier, I999] s'est penché sur les usages contemporains des seconds prénoms. Ces prénoms, il le constate, sont choisis assez souvent en référence à un membre de la famille. Ceci explique leur caractère désuet : ils ne manifestent pas l'appartenance à une génération, mais l'appartenance à une ou plusieurs lignées. Après avoir recueilli les prénoms d'environ 3000 personnes (principalement ses étudiants), l'anthropologue remarque que « $56,8 \%$ des [seconds] prénoms donnés à des filles rattachent celles-ci à leurs parents maternels tandis que 57,4\% de ceux qui sont donnés à des garçons relient ceux-ci à leurs parents paternels. Le fait que le biais parallèle ait la même intensité pour les filles et les garçons peut être considéré comme un indice du caractère égalitaire des rapports entre les deux familles alliées» [Vernier, I 999, p. I I 2-I I 3]. Et si l'enfant porte plusieurs prénoms d'origine familiale, alors ses prénoms font référence, pour 3 cas sur 4 , aux deux lignées. Un principe d'alternance guide les affiliations symboliques : si une lignée est mentionnée, l'autre le sera aussi.

Les prénoms multiples introduisent donc de la souplesse dans les contraintes que les individus se donnent. Ils ne servent d'ailleurs pas qu'à rattacher un individu à ses (grands-)parents. Un quart des bacheliers porteurs de seconds prénoms portent des prénoms qui sont à la mode au moment de leur naissance. Là, ce ne sont pas les prénoms des parents qui sont choisis pour être transmis. Le second prénom (et, éventuellement, le troisième ou le quatrième) est un prénom que les parents appréciaient... mais pas suffisamment pour s'accorder à le mettre en première position. $\mathrm{La}$ " liste des prénoms »- sur laquelle discutent les parents pour établir un consensus à la naissance - se retrouve objectivée dans les prénoms donnés à l'état civil.

Mais ces prénoms, dans la vie quotidienne, restent invisibles : le prénom d'usage est le premier prénom.

\section{Quand le prénom d'usage est différent du pre- mier prénom}

Parfois, le prénom d'usage n'est pas le premier prénom. Pour diverses raisons, une personne est appelée d'un autre prénom. Dans nos sociétés contemporaines, prises dans une « morale d'état civil» qui localise une partie de la vérité des individus dans l'état civil [Foucault, I969, p.28], porter un autre prénom met en question l'authenticité de l'individu. Afin de mieux comprendre cette idée, tour- 
nons notre regard vers le passé récent. Les ethnologues du monde rural français ont longtemps souligné les pratiques de « plurinomination» [Segalen, I 980; Zonabend, I977]. "La mère Raymond, c'était la Michelle, mais son vrai nom c'était Simone » : au baptème, au mariage, à la mort de l'époux, après une grande épreuve... le nom d'usage pouvait changer, sans que l'authenticité des personnes soit remise en cause. Dans ces sociétés d'interconnaissance, les catégories d'état civil nétaient utilisées que si elles étaient en accord avec les habitudes. En cas de mariage « en gendre " (quand le gendre vient habiter dans la maison du père de son épouse), le nom habituel pouvait être le nom de la maison (c'est à dire celui de son épouse). Ainsi «chaque nom est spécifique d'une phase de la vie, d'un statut social et d'un interlocuteur particulier, au point que si on demande à quelqu'un "comment vous appellez-vous?”, il peut répondre : “ça dépend”!» [Fine \& Ouellette, 2005, p.r 9]

Aujourd'hui, et de plus en plus, ce sont les catégories d'état civil qui informent ce que l'on peut connaître d'une personne. À la question «Comment tu t'appelles?", il n'est plus vraiment possible de répondre "ça dépend»: nous avons intégré à la vie quotidienne des outils forgés pour l'administration " à distance » des individus, pour leur identification hors des situations d'interconnaissance. Cela ne fait pas disparaître la plurinomination, mais la rend problématique. Cela transforme un problème d'identification en problème identitaire.

Prendre un prénom d'usage peut alors s'avérer problématique, ce dont il est possible de se rendre compte en étudiant, par exemple, les demandes de changement de prénom ${ }^{\mathbb{T}}$, qui montrent comment « identification » et « identité personnelle » sont aujourd'hui liées. La tension entre ces deux facettes du prénom naît du « contrôle de l'information » nécessaire à certains individus pour que leur stigmate, cet attribut discréditable, reste inconnu (pour reprendre le vocabulaire développé par Goffman dans Stigmate [Goffman, 1975]. Lune des techniques de contrôle est de « diviser le monde en deux groupes » : «l'un, nombreux, auquel il ne révèle rien, et l'autre, restreint, auquel il dit tout et dont il espère le soutien».

Développons cette idée à partir d'un exemple concret. Monsieur X, né vers I 975 demande en 2003 à changer de prénom. À la naissance, il avait été nommé "Johnny, Camille, Philippe », mais il a progressivement commencé à utiliser le prénom Philippe, trouvant Johnny ridicule. Â l'audience, le conseil de M. X a, en présence de son client, exposé que celui-ci présentait un intérêt à agir devant la persistance des administrations à l'appeler Johnny [plutôt que ses autres prénoms] et justifiait d'un intérêt légitime eu égard au ridicule dudit prénom, aux problèmes psychologiques que celui-ci génère et au fait que son entourage le connaisse sous le nom de Philippe.

On trouve, dans la partie de l'arrêt de la cour cherchant à évaluer "l'intérêt

I. Depuis I993, la demande s'adresse au juge aux affaires familiales. Environ 3000 personnes demandent chaque année à changer de prénom. 
légitime”, ceci :

"M. Xprouve:

d'une part qu'il est usuellement prénommé Philippe, et depuis longtemps, (contrat de travail, bulletins de salaire, correspondance Société Générale, attestations), ce qui dépasse la simple convenance personnelle,

d'autre part, qu'il a honte d'avouer son premier prénom Johnny, qu'il cherche à cacher, au point que celui-ci constitue pour lui une véritable entrave d'ordre social: hantise de la découverte de son premier prénom par ceux qui le connaissent sous celui de Philippe, impossibilité de réunir les personnes le connaissant sous le seul prénom de Philippe et celles qui connaissent son secret (attestation [suivent les noms des attestants]), évitement d'avoir à sortir ses papiers officiels en présence d'un tiers (courrier $Y$, psychothérapeute)

enfin que sur le plan psychologique, la relation qu'il entretien avec son prénom Johnny, qu'il juge ridicule et non respectable, inévitablement lié à celui choisi par un chanteur au succès médiatique répandu, prend un caractère traumatique, quand bien même $M$. X est par ailleurs équilibré et sensé [...]»

La cour d'appel autorisera M. X à s'appeler désormais Philippe, et à rapprocher ainsi les deux mondes séparés qu'il avait constitués. C'est en partie parce qu'il est, aujourd'hui, plus difficile de se faire appeler comme on le souhaite que la Cour a autorisé Johnny à devenir Philippe : les administrations — et, plus généralement toutes les institutions qui se sont « branchées » sur les outils étatiques d'identification des citoyens - ont une grande inertie et ne changent pas leurs pratiques de nomination en fonction des souhaits des administrés.

\section{Conclusion}

J'ai abordé ici deux éléments très différents, le deuxième prénom et le prénom d'usage, qui ont la particularité d'être en tension avec le prénom de l'état civil, celui par lequel, habituellement, nous sommes connu. Ces deux éléments soulignent combien le prénom est une trace ambiguë, " inscrite, objectivement et subjectivement (par les donneurs) dans plusieurs registres à la fois » [Bozon, ı987, p.95]. Loin d'être un simple matricule, un numéro d'identification comme le numéro de sécurité sociale, le prénom inscrit dans son porteur toute une série d'éléments : les souhaits des parents, l'image qu'ils veulent se donner, la famille qu'il aimeraient créer...

\section{Bibliographie}

Besnard, P. [I 979], Pour une étude empirique du phénomène de mode dans la consommation des biens symboliques : le cas des prénoms. Archives européennes de 
sociologie, $20, \mathrm{p} \cdot 343-35 \mathrm{I}$.

Bozon, M. [1987], Histoire et sociologie d'un bien symbolique, le prénom. Population, I, p.83-98.

Coulmont, B. [201 I], Sociologie des prénoms, Paris : La Découverte.

Daumas, P. [2003], Familles en Révolution. Vie et relations familiales en Île de France, changements et continuités (I775-I825), Rennes : Presses universitaires de Rennes.

Dupâquier, J., Pelissier, J.-P. \& Rébaudo, D. [1987], Le Temps des Jules. Les prénoms en France au XIXe siècle, Paris : Editions Christian.

Fine, A. \& Ouellette, F.-R. éd. [2005], Le Nom dans les sociétés occidentales contemporaines, Toulouse : Presses universitaires du Mirail.

Foucault, M. [1 969], L'Archéologie du savoir, Paris : Gallimard.

Goffman, E. [1975], Stigmate. Les usages sociaux du handicap., Paris : Minuit.

Lieberson, S. [2000], A Matter of Taste. How Names, Fashions, and Culture Change, New Haven et Londres : Yale University Press.

Perouas, L. et al. [1984], Léonard, Marie, Jean et les autres. Les prénoms en Limousin depuis un millénaire, Paris : Editions du Centre national de la recherche scientifique.

Segalen, M. [1980], Le nom caché. La dénomination dans le pays bigouden sud. L'Homme, 2o[4], p.63-76.

Vernier, B. [1 999], Le visage et le nom : Contribution à l'étude des systèmes de parenté, Paris : Presses Universitaires de France.

Vernier, B. [1998], Prénom et ressemblance. Appropriation symbolique des enfants, économie affective et systèmes de parenté. Dans A. Fine, éd. Adoptions. Ethnologie des parentés choisies. Paris : Éditions de la MSH, p. 97-I I 9.

Zonabend, F. [1977], Pourquoi nommer? (Les noms de personnes dans un village français : Minot-en-Châtillonais). Dans C. Lévi-Strauss, éd. L'Identité. Paris : Grasset, p. 257-279. 